\title{
ACTIVATION OF LYMPHOCYTE PROLIFERATION BY BORONATE-CONTAINING POLYMER IMMOBILISED ON SUBSTRATE: THE EFFECT OF BORON CONTENT ON LYMPHOCYTE PROLIFERATION
}

\author{
Hidenori Otsuka ${ }^{1,2,3}$, Takeshi Ikeya ${ }^{4}$, Teruo Okano ${ }^{5}$ and Kazunori Kataoka $a^{2,6,7 *}$ \\ 'Department of Applied Chemistry, Faculty of Science, Tokyo University of Science, 1-3 Kagurazaka, Shinjuku-ku, \\ Tokyo 162-8601, Japan \\ ${ }^{2}$ Department of Materials Engineering, Graduate School of Engineering, The University of Tokyo, 7-3-1 Hongo, \\ Bunkyo-ku, Tokyo 113-8656, Japan \\ ${ }^{3}$ Biomaterials Center, National Institute for Materials Science (NIMS), 1-1 Namiki, Tsukuba, Ibaraki 305-0044, \\ Japan \\ ${ }^{4}$ Department of Materials Science, Tokyo University of Science, Noda 278-8510, Japan \\ ${ }^{5}$ Institute of Advanced Biomedical Engineering and Science, Tokyo Women's Medical University, 8-1 Kawada-cho, \\ Shinjuku-ku, Tokyo 162-8666, Japan \\ ${ }^{6}$ Division of Clinical Biotechnology, Center for Disease Biology and Integrative Medicine, Graduate School of \\ Medicine, The University of Tokyo, 7-3-1 Hongo, Bunkyo-ku, Tokyo 113-8656, Japan \\ ${ }^{7}$ Center for NanoBio Integration, The University of Tokyo. 7-3-1 Hongo, Bunkyo-ku, Tokyo. 113-8656, Japan
}

\begin{abstract}
This study demonstrates that boronic acid-containing polymers coated onto solid support function as synthetic mitogens for mouse lymphocytes. The polymer was synthesized by radical copolymerization of 3acrylamidophenylboronic acid with dimethylacrylamide (poly(AAPBA-DMAA)). The boronic acid in the trigonal form in the copolymer activated lymphocytes, probably by crosslinkage to glycoprotein moieties on the plasma membrane surface, as in the case of lectin stimulation. A higher concentration of phenylboronic acid on the copolymer surface resulted in greater activation of lymphocytes, suggesting that the number of phenylboronic acid residues per unit area may be a crucial factor in lymphocyte proliferation. The proliferative response of lymphocytes was also affected by the surface wettability, probably due to a difference in the flexibility of polymer strands at the cell-polymer interface.
\end{abstract}

Key Words: Lymphocyte, phenylboronate, boronic acidcontaining polymer, artificial lectin, mitogen.

*Address for correspondence:

Kazunori Kataoka

Department of Materials Engineering,

Graduate School of Engineering,

The University of Tokyo, 7-3-1 Hongo,

Bunkyo-ku, Tokyo 113-8656, Japan

Telephone Number:+81-3-5841-7138,

FAX Number:+81-3-5841-7139

E-mail: kataoka@bmw.t.u-tokyo.ac.jp

\section{Introduction}

Lymphocytes consist of various subpopulations with distinctive functions, which play important roles in immune responses (Marchalonis, 1988). Activation and proliferation of these subpopulations can be achieved by treating them with mitogens. The best-known group of mitogens are sugar-binding proteins called lectin. Lectins have been widely used for cell separation and functional analysis owing to their specific binding properties to a particular sugar sequence expressed on the surface of the plasma membrane (Sharon and Lis, 1972, 1998). Lectin treatment occasionally induces lymphocytes with high anti-tumor activity, and thus it is expected to be useful for cancer therapy (Mulé et al., 1984; Mulé and Rosenberg, 1985). However, lectins of plant origin have antigenicity in humans (Franz et al., 1983; Kulkarni et al., 1998; Leist and Wendel, 1998), and their stability are not always adequate for use in immunotherapy and medical engineering, including tissue engineering and drug delivery (Kijne, 1996). These problems may be resolved through the development of a completely synthetic polymer with lectin-like function (Miyazaki et al., 1993).

We previously synthesized a novel water-soluble polymer with lectin-like function (artificial lectin) by introducing phenylboronates, as sugar-recognizing moieties, into the side-chain of poly-N,Ndimethylacrylamide (Miyazaki, 1993). Phenylboronates were chosen because they can easily form reversible covalent bonds with polyol compounds, including sugars (Conner and Bulgrin, 1967; Barker et al., 1973). At physiological $\mathrm{pH}$, phenylboronates form an appreciably stable complex with sialic acid (Neu5Ac), a characteristic anionic carbohydrate on the surface of the plasma membranes (Otsuka et al., 2003). A prepared polymer induced appreciable proliferation of murine splenic lymphocytes with increased expression of IL-2 receptor on their surface, presumably due to the selective binding to Neu5A components on the lymphocyte membrane (Uchimura et al., 2001). Increased proliferation of lymphocytes showing cytotoxicity against YAC-1 cells 
was achieved by concurrent addition of IL- 2 with the boronate-containing polymer in the medium, suggesting that this polymer may be an effective immuno-adjuvant for the induction of lymphokine-activated killer (LAK) cells (Uchimura et al., 2001).

Moreover, compared with its soluble form, immobilized natural lectin on solid matrix induced even greater proliferation of cells, including lymphocytes (Greaves and Bauminger, 1972). For example, a polymer substrate with immobilized Ulex I lectin was reported to have a high ability to induce adhesion and successive proliferation of vascular endothelial cells (Parhizgar et al., 1987), suggesting that lectin-immobilized substrate may be useful in the field of tissue engineering. Enhanced uptake of particles by gastrointestinal epithelial cells has been achieved by immobilizing a certain group of lectins (Tomato, Mycoplasma) on the particle surface (Irache et al., 1994), which may become a useful approach for oral delivery of vaccine and other physiologically active compounds. Inspired by these results, we performed a systematic study of the interaction of cells, including lymphocytes, with substrates coated with phenylboronatecontaining polymer, to obtain an important basis for the development of boronate-based solid-support which might be useful for the modulation of cellular functions (Kataoka et al., 1989; Jozefowicz and Jozefowicz, 1990). When bovine aortic endothelial cells were cultured on substrate coated with phenylboronate-containing polymer they adhered together and proliferated, spontaneously forming a capillary structure (Aoki et al., 1995).

Here, we report the use of a boronic acid-containing solid substrate for the activation and proliferation of lymphocytes through the binding to carbohydrates expressed on their plasma membrane. The substrate was composed of N,N-dimethylacrylamide (DMAA) and 3acrylamidophenylboronic acid (AAPBA). An important aim of the study was clarification of the effects of the surface properties of the substrate, including the surface boron concentration and wettability, on the proliferative response of lymphocytes.

\section{Experimental}

\section{Materials}

DMAA (Wako Pure Chemical Co. Ltd., Tokyo, Japan) was purified by distillation under reduced pressure. AAPBA (Wako) and 2, 2'-azobis(2, 4-dimethylvaleronitrile) (V65; Wako) were recrystallized from ethanol and dried thoroughly in vacuo at room temperature. NPhenylacrylamide (PA; Polyscience Inc., Warrington, PA, USA) was obtained commercially and used without further purification. Other reagents and solvents were purified by standard methods.

\section{Preparation of Polymer Samples}

Copolymers of DMAA with AAPBA(DB copolymer) with varying composition were prepared by radical copolymerization as follows: various ratios of DMAA and AAPBA were dissolved in ethanol, and V-65 was added to initiate copolymerization. The reaction mixture was stirred in a sealed glass ampoule in vacuo at $40^{\circ} \mathrm{C}$ for 30 min. Polymerization was quenched by pouring the reaction mixture into excess diethyl ether. The precipitated DB was filtered and dried thoroughly in vacuo. As a control polymer sample without phenylboronic acid moieties, copolymer DP was prepared by radical copolymerization of DMAA with PA, following a procedure similar to that used to prepare $\mathrm{DB}$ but at a reaction temperature of $45^{\circ} \mathrm{C}$ and with different concentrations of the monomers and initiator (Ikeya et al., 1998).

Compositions of DB and DP were determined by ${ }^{1} \mathrm{H}$ NMR (EX-400, JEOL Ltd., Tokyo, Japan) in DMSO-d at $80^{\circ} \mathrm{C}$. DB and DP were designated as DB-X and DP-X, where $\mathrm{X}$ is the mol\% of AAPBA and PA, respectively. Their weight-averaged molecular weights were determined by static light scattering (DLS-700, Otsuka Electronics Co. Ltd., Osaka, Japan) in ethanol.

\section{Determination of Surface Boron Contents of DB-X Film by X-ray Photoelectron Spectroscopy (XPS)}

The elemental composition of the surface of a DB-X film on polystyrene disks (diameter: $8 \mathrm{~mm}$ ) were determined using XPS to quantify the phenylboronic acid moieties in the outermost layer of the films. The samples were prepared by casting DB-X polymers in ethanol onto the polystyrene disks. Spectra were acquired using an AXIS-MS electron spectrometer (Kratos Analytical Ltd., Manchester, UK), and the take-off angle was $90^{\circ}$. Five survey scans and twenty detailed scans of C1s and B1s were recorded for each sample. The area ratio of $\mathrm{B} 1 \mathrm{~s}$ peak (binding energy: $200 \mathrm{eV}$ ) to $\mathrm{C} 1 \mathrm{~s}$ peak (binding energy: $295 \mathrm{eV}$ ) (B/C ratio) was used as an index of the boron content on the copolymer surface.

Contact angle measurement (Otsuka et al., 2000a,b)

The variation of static wettability of the surface covered with $0.5 \%$ DB and DP copolymers by buffered solutions

Table 1. Characterization of polymer samples.

\begin{tabular}{|c|c|c|c|c|c|c|c|c|}
\hline \multirow[t]{2}{*}{ code } & \multicolumn{3}{|c|}{$\begin{array}{l}\text { feed composition } \\
\text { (molar ratio) }\end{array}$} & \multirow[t]{2}{*}{$\begin{array}{l}\text { yield } \\
(\%)\end{array}$} & \multicolumn{3}{|c|}{$\begin{array}{l}\text { molar composition of } \\
\text { obtained polymers }\end{array}$} & \multirow[t]{2}{*}{$\begin{array}{l}\mathrm{Mw} b) \\
\left(\mathrm{X} 10^{5}\right)\end{array}$} \\
\hline & DMAA & AAPBA & PA & & DMAA & AAPBA & $\mathrm{PA}$ & \\
\hline DB-17.5 & 0.875 & 0.125 & - & 30.3 & 0.825 & 0.175 & - & 0.9 \\
\hline DB-33 & 0.7 & 0.3 & - & 70.4 & 0.67 & 0.33 & - & 3.0 \\
\hline DB-55 & 0.5 & 0.5 & - & 81.5 & 0.45 & 0.55 & - & 4.4 \\
\hline DP-37 & 07 & - & 0.3 & 197 & 0.63 & & 0.37 & 0.6 \\
\hline
\end{tabular}

a) Copolymer composition was determined by ${ }^{1} \mathrm{H}-\mathrm{NMR}$

b) Mw was determined by static light scatting in $\mathrm{EtOH}$. 
in the $\mathrm{pH}$ range of 5-11 was evaluated using a contact angle meter (CA-W: Kyowa Kaimen Kagaku Co. Tokyo, Japan). The buffers were $\mathrm{CH}_{3} \mathrm{COOH} / \mathrm{NaOH} / \mathrm{NaCl}(\mathrm{pH} 5)$, phosphate buffer solution $(6.0<\mathrm{pH}<8.0)$, sodium $p$ phenosulfonate $/ \mathrm{NaOH}$ buffer solution $(8.0<\mathrm{pH}<9.0)$, and $\mathrm{NaHCO}_{3} / \mathrm{NaOH} / \mathrm{NaCl}$ buffer solution (>pH9.0), all with an ionic strength of 0.15 , maintained by adding an appropriate amount of $\mathrm{NaCl}$. The captive bubble technique was used, whereby the sample film was immersed in water maintained at $25^{\circ} \mathrm{C}$ and a small air bubble was placed under the film using a curved needle (Fig. 3). The contact angle of each film sample was measured at 15 or more spots, and the values were averaged.

\section{Lymphocyte Preparation}

Mouse lymphocytes were obtained under sterilized conditions from spleens of 5-week-old male AKR mice (Sankyo Laboratories Co. Ltd., Tokyo, Japan) sacrificed by dislocation of the cervical vertebrae (Miyazaki et al., 1993). The spleens were immersed immediately into 25 mM HEPES (N-2-hydroxyethyl-N'-2-sulfoethylpiperazine)-buffered RPMI 1640 medium in Petri dishes. The lymphocytes were released by flushing the spleens several times with RPMI 1640 medium using a 10-ml disposable plastic syringe fitted with a 26-gauge needle. The lymphocyte suspension was filtered through a $70-\mu \mathrm{m}$ mesh nylon net to remove debris, and centrifuged at $200 \mathrm{~g}$ for $5 \mathrm{~min}$ at room temperature. The lymphocyte pellets were then mixed gently with Tris-buffered $0.83 \%(\mathrm{w} / \mathrm{w})$ $\mathrm{NH}_{4} \mathrm{Cl}$ to lyse contaminating erythrocytes (Boyum, 1968), and washed twice with RPMI 1640 medium. The number of lymphocytes in the suspension was counted using a Coulter counter (model ZM, Beckman Coulter Inc., Fullerton, CA, USA), and adjusted to the desired cell concentration by addition of $25 \mathrm{mM}$ HEPES-buffered RPMI 1640 medium.

\section{Lymphocyte Proliferation Assay}

The copolymer dissolved to a certain concentration in ethanol was used to prepare the polymer coating on the inner surface of a flat-bottomed multi-well plate (Falcon 3042, Becton-Dickinson Co. Ltd., Franklin Lakes, NJ, USA). Each well was treated with the polymer solution and dried under argon at room temperature for 2 days and then in vacuo at $45^{\circ} \mathrm{C}$ for 1 day. Lymphocytes were suspended in $25 \mathrm{mM}$ HEPES-buffered RPMI 1640 medium supplemented with $10 \%$ fetal bovine serum (FBS) (Dainippon Pharmaceutical Co. Ltd., Osaka, Japan) at $2 \times 10^{6}$ cells $/ \mathrm{ml}$, and $200 \mu \mathrm{l}$ of this suspension was plated into a polymer coated multi-well plate. The lymphocytes were cultured at $37^{\circ} \mathrm{C}$ for $45 \mathrm{~h}$ in a humidified $5 \% \mathrm{CO}_{2}$ atmosphere. Then, $20 \mu \mathrm{l}$ of ${ }^{3} \mathrm{H}$-thymidine (AmershamPharmacia Biotech, Uppsala, Sweden) was added to the suspension and the lymphocytes cultured for a further $3 \mathrm{~h}$. The lymphocytes were harvested onto a glass filter (Skatron Instrument Inc., Sterling, VA, USA), and their radioactivity was measured by liquid scintillation (Aloka Co. Ltd., Tokyo, Japan) using a scintillation cocktail (27 $\mathrm{mM}$ of 2, 5-diphenyloxazole and $1.1 \mathrm{mM}$ of 4-bis(2phenyloxazoyl)benzene in toluene).

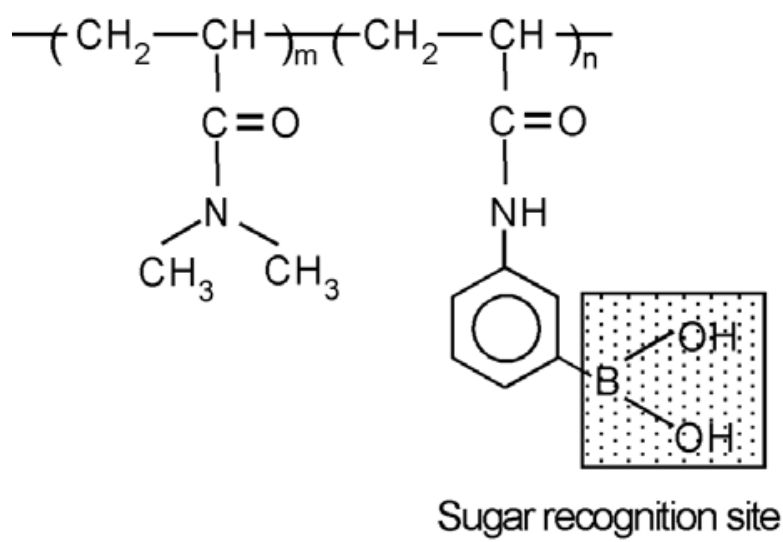

poly(AAPBA-DMAA) (DB-X)<smiles>CCCCCC(CC)C(=O)N(C)C</smiles>

poly(PA-DMAA) (DP-X)

Figure 1. Structural formulas of copolymers of N,Ndimethylacrylamide (DMAA) with 3acrylamidophenylboronic acid (AAPBA), shown as poly(AAPBA-DMAA) (DB copolymer), and DMAA with N-Phenylacrylamide (PA), shown as poly(PADMAA) (DP copolymer). The value of $x$ denotes the molar composition of AAPBA for poly(AAPBADMAA) and PA for poly(PA-DMAA), respectively.

\section{Results and Discussion}

\section{Characterization of Substrata Coated with Boronate-containing Copolymer}

Fig. 1 shows the structural formulae of DB and DP. Table 1 lists their weight-averaged molecular weights, chemical structure, and compositions. The contents of AAPBA and DP units in the copolymer were controlled so as to be waterinsoluble in the experimental conditions.

The elemental binding states and compositions of polystyrene surfaces coated with DB copolymer were examined by survey scans and high resolution $\mathrm{C} 1 \mathrm{~s}$ and B1s of XPS analysis, and the results are summarised in Fig. 2 as a function of the coating concentration of the copolymer with the ratio of core-level photoelectron emissions for $\mathrm{B} 1 \mathrm{~s}$ to $\mathrm{C} 1 \mathrm{~s}$. This ratio is used as an index of the boron content on the copolymer surface. Obviously, the ratio increases with coating concentration and then reaches plateau value in the range of 4 to 6 irrespective of 
the copolymer composition. The calculated $\mathrm{B} 1 / \mathrm{C} 1$ ratios based on sensitivity factor $(\mathrm{B}: \mathrm{C}=0.49: 1)$ were 1.51 for $\mathrm{DB}$ 17.7, 2.49 for DB-33, and 3.68 for DB-55, respectively, suggesting that phenylboronic acid moieties tend to be enriched at the surface for all coatings, probably owing to their hydrophobic character. The surface boron content of DB increases with PBA composition.

$\mathrm{pH}$-dependent change in contact angle of the charged surface determined by contact angle titration provides data on the dissociation state and concentration of charged functional groups on the surface, and correlates with material properties, at the macroscopic level, such as wettability and adhesion strength (Holmes-Farley et al., 1985; Holmes-Farley and Whitesides, 1986). Contact angle titration results have also been correlated with the degree of ionization determined directly by ATR-IR spectroscopy (Holmes-Farley et al., 1985), fluorescence spectroscopy (Holmes-Farley and Whitesides, 1986), and conventional titration (Holmes-Farley et al., 1985). Holmes-Farley et al. (1985) reported that the contact angle of water on polyethylene film derivatized with ionizable carboxylate groups changes systematically with the $\mathrm{pH}$ of the water, correlating nicely with the ionization degree of the carboxylate groups. However, this study was performed using dry films, and thus, the measured interface may not be in thermodynamic equilibrium with the water phase, resulting in hysteresis. To avoid this effect, the wettability of the boronated surface was estimated in buffer $(I=0.15)$, providing information regarding the boronic acid content and its dissociation equilibrium on the surface at a given $\mathrm{pH}$. Table 2 summarizes the contact angle titrations on the film samples coated with DB and DP copolymers, respectively, as a function of $\mathrm{pH}$. As DB-17.5 was slightly water-soluble under alkaline conditions, contact angle titration was performed for DB-33 and DB-55. With the captive bubble technique shown in Fig. 3, the higher contact angle $(\theta)$ corresponds to the increased hydrophilicity. Table 2 clearly indicates that surfaces containing ionizable boronate groups (DB-33, DB-55) undergo an appreciable change in contact angle with $\mathrm{pH}$ : a higher contact angle is associated with higher $\mathrm{pH}$. This is due to increased ionization of the boronate with higher $\mathrm{pH}$, which induces increased water adsorption onto the polar and charged tetrahedral boronate group. The work

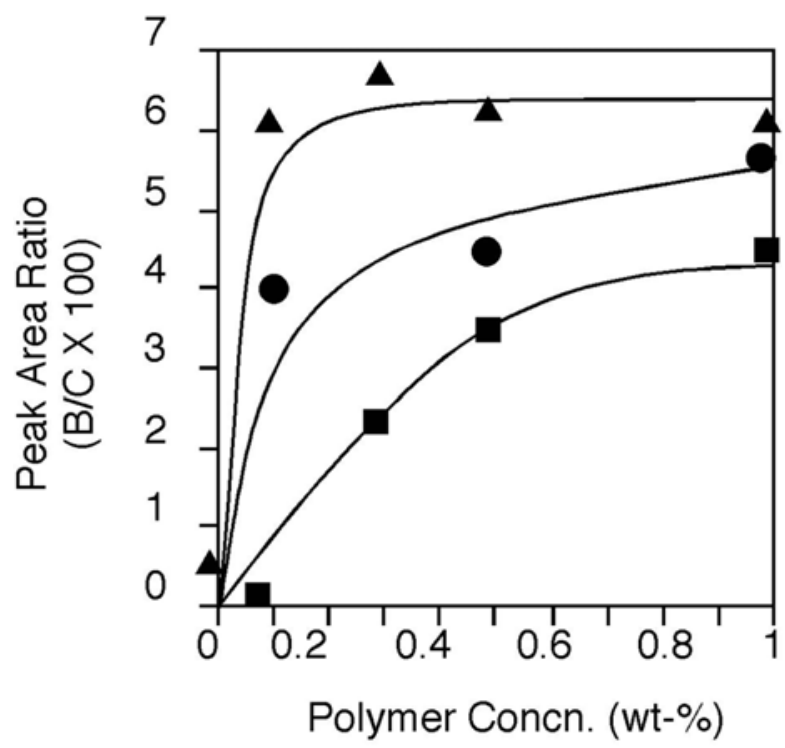

Figure 2. Phenylboronic acid content on the substrate coated with poly(AAPBA-DMAA) (DB copolymer) examined by survey scans and high resolution $\mathrm{C} 1 \mathrm{~s}$ and $\mathrm{B} 1 \mathrm{~s}$ of XPS analysis, which is summarised as a function of the coating concentration of the copolymer with the ratio of core-level photoelectron emissions for B1s to C1s. ( $\bullet$ DB-17.5, (○) DB-33, and ( $\mathbf{\Delta})$ DB-55.

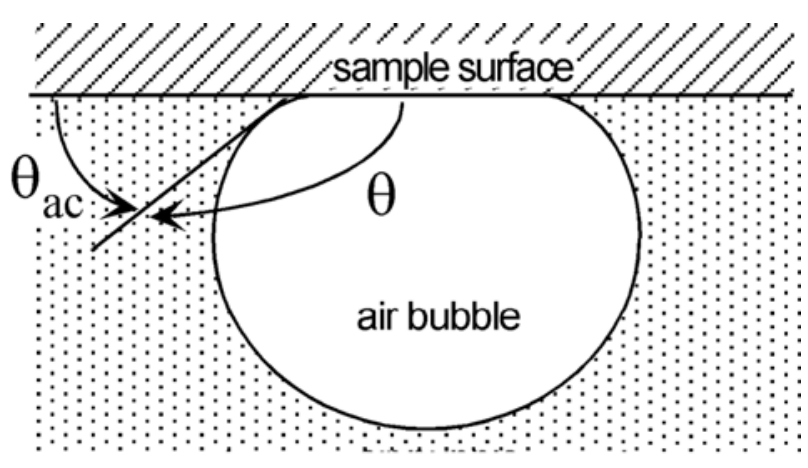

Figure 3. Schemaic representation of contact angle measurement in water. $\theta$ (degree) is the angle between the solid surface and the air bubble interface measured inside the bubble. $\theta$ is related to the actual contact angle $\theta_{\text {ac }}$ by $\theta=180-\theta_{\text {ac }}$. The higher contact angle $(\theta)$ corresponds to the increased hydrophilicity (smaller value of $\theta_{\mathrm{ac}}$ ).

Table 2. Contact angle titration for interfaces with and without phenylboronic acid groups.

\begin{tabular}{|c|c|c|c|c|c|c|}
\hline \multirow[b]{2}{*}{$\mathrm{pH}$} & \multicolumn{2}{|c|}{ DP-37 } & \multicolumn{2}{|c|}{ DB-33 } & \multicolumn{2}{|c|}{ DB-55 } \\
\hline & $\theta$ & $\cos (180-\theta)$ & $\theta$ & $\cos (180-\theta)$ & $\theta$ & $\cos (180-\theta)$ \\
\hline 4 & 117.7 & 0.46 & 119.5 & 0.49 & 110.5 & 0.35 \\
\hline 5 & 118.5 & 0.48 & 121.1 & 0.52 & 111.3 & 0.36 \\
\hline 6.2 & 118.8 & 0.48 & 124.4 & 0.56 & 113.0 & 0.39 \\
\hline 7.4 & 118.9 & 0.48 & 135.7 & 0.72 & 123.6 & 0.55 \\
\hline 8.6 & 117.1 & 0.46 & 143.2 & 0.80 & 148.4 & 0.85 \\
\hline 9.8 & 118.8 & 0.48 & 150.0 & 0.87 & 157.9 & 0.93 \\
\hline 11 & 118.8 & 0.48 & 151.0 & 0.87 & 158.5 & 0.93 \\
\hline
\end{tabular}




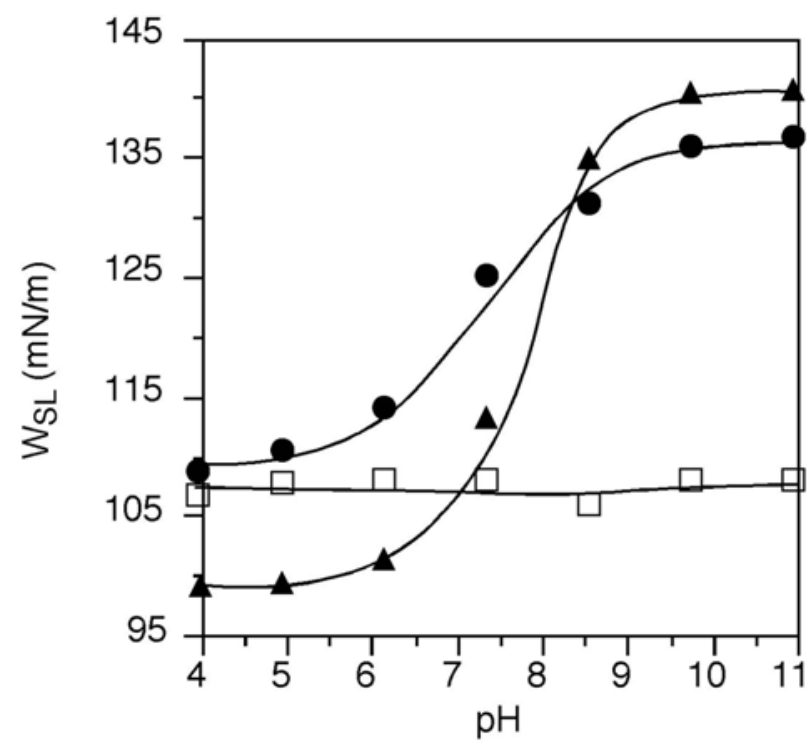

Figure 4. Contact angle titration for interfaces with and without phenylboronic acid groups, showing the work of adhesion between the contacting solid--liquid interface, $\mathrm{W}_{\mathrm{SL}}$, calculated as a function of $\mathrm{pH}$. ( $) \mathrm{DB}$ 33, (A) DB-55, and ( $\square$ ) DP-37.

of adhesion between the contacting solid-liquid interface, $\mathrm{W}_{\mathrm{SL}}$, was then calculated as a function of $\mathrm{pH}$ to get more insight into the system (Fig. 4). As shown in eqs. (1) and (2), $\mathrm{W}_{\mathrm{SL}}$ includes a substantial contribution from both polar and hydrogen bonding components.

$W_{s L}=\gamma_{L}(1+\cos \theta) \quad$ (Young - Dupre equation)

$=\gamma_{L}+\gamma_{S}-\gamma_{S L}$

$=2 \sqrt{\gamma_{L}^{d} \gamma_{S}^{d}}+2 \sqrt{\gamma_{L}^{p} \gamma_{S}^{p}}+2 \sqrt{\gamma_{L}^{h} \gamma_{S}^{h}} \quad$ (geometric mean approximation )

(2)

$=W_{S L}^{d}+W_{S L}^{p}+W_{S L}^{h}$

where $\gamma^{\mathrm{d}}, \gamma^{\mathrm{p}}$, and $\gamma^{\mathrm{h}}$ are components of the surface free energy $\gamma$, arising from the dispersion force, the polar force, and the hydrogen-bonding force, respectively. Dipole and hydrogen bonding characteristics should be important because the hydration of phenylboronic acid through ionization on the DB surface is the main factor in wettability, which influences lymphocyte interaction. Fig. 4 shows that the $\mathrm{W}_{\mathrm{SL}}$ of surfaces coated with $\mathrm{DB}$ copolymers is considerably dependent on $\mathrm{pH}$, which is in agreement with the titration curve for 3propionamidophenylboronic acid; PAPBA, a model compound of AAPBA units in DB copolymer (Hisamitsu, 1997). The change in $\mathrm{W}_{\mathrm{SL}}$ with $\mathrm{pH}$ was more significant for DB-55 than for DB-33, reflecting the higher concentration of boronate on the former surface (Fig. 2). This was also suggested by the higher $\mathrm{W}_{\mathrm{SL}}$ value for DB55 at $\mathrm{pH} 8.6$, which is the $\mathrm{pKa}$ of the corresponding monomer (PAPBA) (Kataoka et al., 1994). On the other hand, the surface coated with DP copolymer as control showed no change in $\mathrm{W}_{\mathrm{SL}}$ as a function of $\mathrm{pH}$. Accordingly, the equilibrium between the neutral phenylboronic acid and the anionic phenylboronate is indeed a key factor in the characteristics of the DB-coated surface.

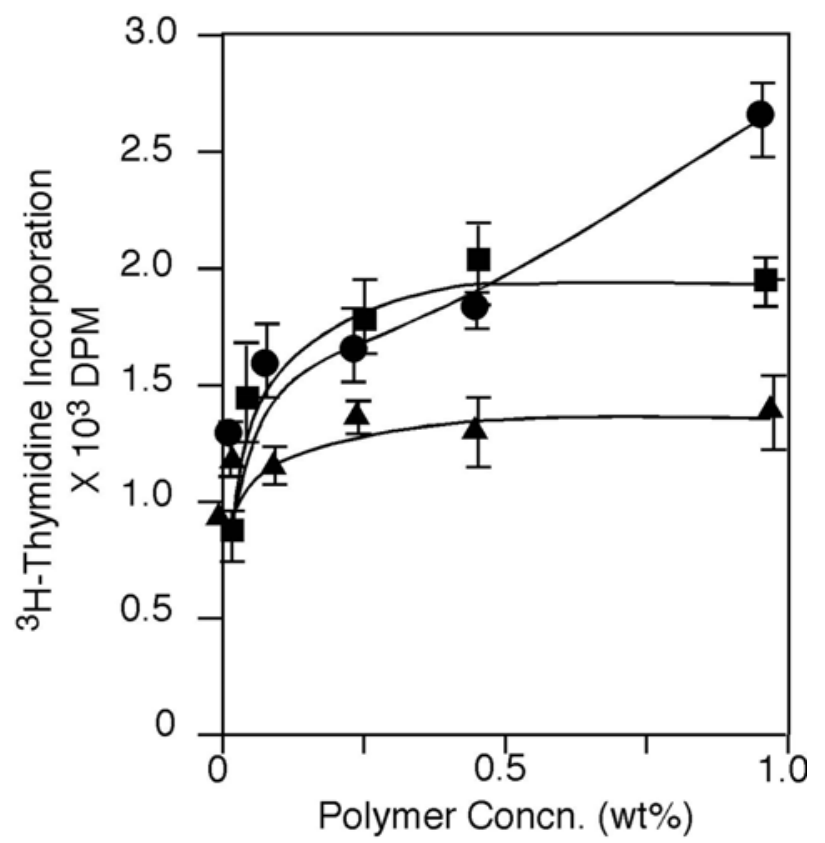

Figure 5. ${ }^{3} \mathrm{H}$ thymidine incorporation of lymphocytes stimulated by poly(AAPBA-DMAA) (DB copolymer) on the substrate. Mouse spleen cells $\left(4.0 \times 10^{5}\right.$ cells/well) were activated with varying concentration of poly(AAPBA-DMAA): ( $\mathbf{a}$ ) DB-17.5, (○) DB-33, and (A) DB-55 on the substrate. Vertical bars represent S.E.M. of triplicate experiments.

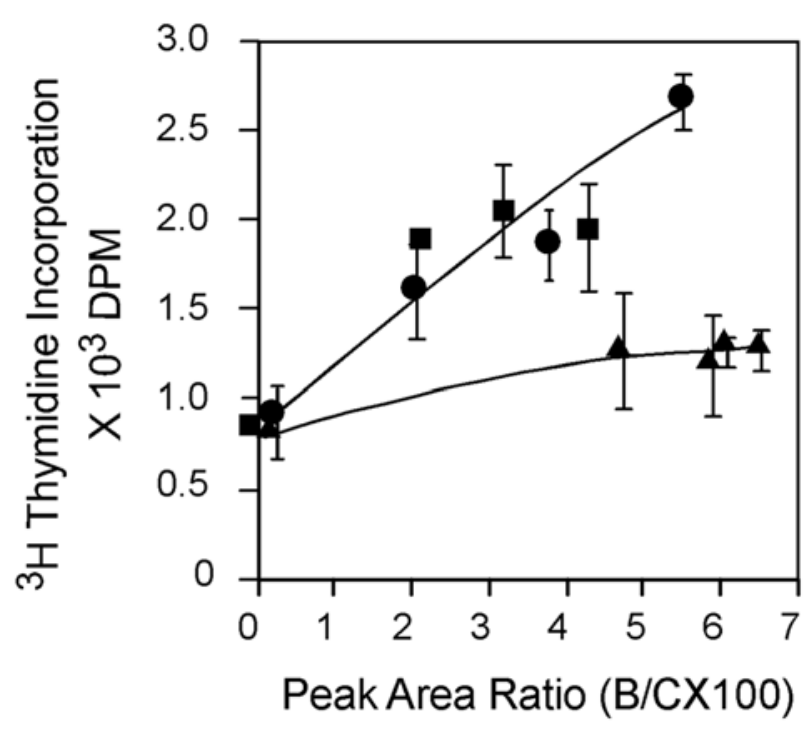

Figure 6. Effect of immobilized phenylboronic acid content on ${ }^{3} \mathrm{H}$-thymidine incorporation of lymphocytes: ( $\mathbf{\square}) \mathrm{DB}-17.5$, ( ) DB-33, and ( $\mathbf{A}) \mathrm{DB}-55$ on the substrate. Immobilized phenylboronic acid content was estimated from $\mathrm{B} / \mathrm{N}$ area ratio in XPS spectra (take-off angle: $90^{\circ}$ ). 


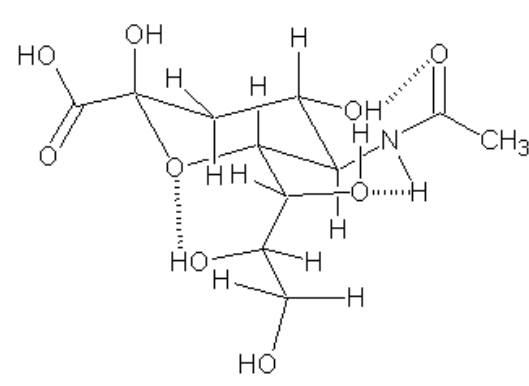

Free NeuAc

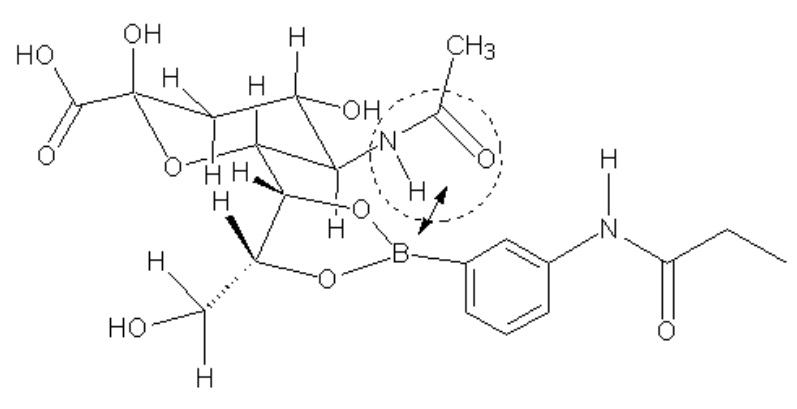

Complex(major form)

Figure 7. Conformational model for the Neu5Ac and the Neu5Ac/PAPBA complex, confirmed from NMR analysis at physiological $\mathrm{pH} 7.4$.

\section{Lymphocyte Proliferation Assay}

The feasibility of DB copolymer-coated substrates stimulating lymphocytes to proliferate was examined by monitoring the ${ }^{3} \mathrm{H}$-thymidine uptake of lymphocytes after $48 \mathrm{~h}$ of cultivation in DB copolymer-coated wells. Figure 5 shows ${ }^{3} \mathrm{H}$-thymidine incorporation into lymphocytes cultured in polystyrene wells coated with DB copolymers with varying boronate content. ${ }^{3} \mathrm{H}$-Thymidine uptake increased with increasing concentration of the DB coating the wells, ${ }^{3} \mathrm{H}$-thymidine uptake levelled off in the range of 0.5-1 wt- $\%$ on surfaces covered with DB-17.5 ( $)$ and 55 (A).

DP copolymer without phenylboronic acid moiety does not induce ${ }^{3} \mathrm{H}$-thymidine uptake by lymphocytes (Uchimura et al., 2001). The essential role of phenylboronate moieties in lymphocyte proliferation was confirmed by our previous study demonstrating appreciable inhibition of boronate-induced lymphocyte proliferation by sorbitol, which is known to have a considerably high binding affinity to phenylborate compounds (Uchimura et al., 2001). Moreover Fig. 5 indicates that the extent of lymphocyte proliferation is dependent on the boron content in the copolymer. To get some insight into this observation, Fig. 5 was replotted as Fig. 6 in which the surface boron content had been determined by XPS.

With increasing amounts of boron on the surface, an appreciable increase in ${ }^{3} \mathrm{H}$-thymidine uptake was observed for DB-17.5 and DB-33 surfaces, while no significant increase in the proliferation of lymphocytes was observed on the DB-55 surface, as shown in Figure 6. Consequently, at the same surface boron concentration, marked difference in the ability to proliferate lymphocytes was observed between the surfaces with low (DB-17.5 and DB-33) and high (DB-55) boron contents. Our previous study confirmed the anomalously high equilibrium constant $(\mathrm{K})$ for Neu5Ac binding to PAPBA at physiological pH7.4 through the formation of the characteristic trigonal borate complexes, as schematically shown in Fig. 7 (Otsuka et al., 2003). Presumably, the appreciably higher proliferation ability of DB-17.5 and DB-33 compared with DB-55 may be correlated to their higher hydrophilic surface at $\mathrm{pH} 7.4$. A less hydrated polymer chain of DB-55 is assumed to have lower ability to access the carbohydrate (Neu5A) moieties on the lymphocytes due to the limited conformational motion. On the other hand, the hydrophilic
DB-17.5 and DB-33 coated surfaces contain higher amounts of associated water to induce chain flexibility, which appreciably contribute to lymphocyte recognition by the borate binding to the carbohydrate moieties on the lymphocyte plasma membrane.

\section{Conclusion}

Solid matrix coated with polydimethylacrylamide partially derived from phenylboronic acid (DB copolymer) was evaluated as a synthetic mitogen of lymphocytes. Phenylboronic acid moiety in the copolymer works as a recognition site of carbohydrates, presumably Neu5Ac, on the surface of lymphocytes through the formation of a stable ester linkage. The amount of substantial phenylboronic acid per unit area seems to be a determining factor in lymphocyte proliferation, and increased amounts of phenylboronic acid results in activation of more lymphocytes. The proliferative response of lymphocytes was further emphasized by increased surface hydrophilicity, probably because increased flexibility of the polymer strands at the interface provide more freedom in spatial matching of polymer-bound boronic acid to carbohydrate moieties expressed on the lymphocyte surface. The improved mitogenic activity by immobilization of the polymer on solid support suggests that lymphocytes may be activated by a column with immobilized phenylboronic acid-derivatized copolymer. This would probably be useful in the field of immunomodulation and cellular engineering, where engineered biomaterials with cell-regulating functions are in strong demand.

\section{Acknowledgements}

This work was supported by the Japan Society for the Promotion of Science, "Research for the Future" Program (JSPS-RFTF96I00201). This work was also supported in part by the Special Coordination Funds for Promoting Science and Technology (16300165) and also supported by Research Promotion Bureau under the contract No. 1783 both from the Ministry of Education, Culture, Sports, Science and Technology (MEXT), Japan. 


\section{References}

Aoki T, Nagao Y, Terada E, Sanui K, Ogata N, Yamada N, Sakurai Y, Kataoka K, Okano T (1995) Endothelial cell differentiation into capillary structures by copolymer surfaces with phenylboronic acid group. J Biomater Sci Polym Ed 7: 539-550.

Barker SA, Chopa AK, Hatt BW, Somers PJ (1973) The interaction of areneboronic acids with monosaccharides. Carbohydr Res 26: 33-40.

Boyum A (1968) Isolation of leucocytes from human blood. A two-phase system for removal of red cells with methylcellulose as erythrocyte-aggregating agent. Scand J Clin Lab Invest 21: Suppl.97: 9-29.

Conner JM, Bulgrin VC (1967) Equilibria between borate ion and some. polyols in aqueous solution. J Inorg Nucl Chem 29: 1953-1961.

Franz H, Kindt A, Eifler R, Ziska P, Benndorf R, Junghahn I (1983) Differences in toxicity and antigenicity between mistletoe lectin I and viscotoxin A 3. Biomed Biochim Acta 42: K21-K25.

Greaves MF, Bauminger S (1972) Activation of T and B lymphocytes by insoluble phytomitogens. Nat New Biol 235: 67-70.

Hisamitsu I (1997) Ph.D. Thesis, Sci Univ Tokyo.

Holmes-Farley SR, Whitesides GM (1986) Fluorescence properties of dansyl groups covalently bonded to the surface of oxidatively functionalized lowdensity pol yet hylene film. Langmuir 2: 266-281.

Holmes-Farley SR, Reamey RH, McCarthy TJ, Deutch J, Whitesides GM (1985) Acid-base behavior of carboxylic acid groups covalently attached at the surface of polyethylene: The usefulness of contact angle in following the ionization of surface functionality. Langmuir 1: 725740 .

Ikeya T, Kataoka K, Okano T, Sakurai Y (1998) Selective adhesion of rat lymphocyte subpopulation on the polymer surface with phenylboronic acid moieties: evaluation by field-flow fractionation/adhesion chromatography (FFF/AC) method. Reac Func Polym 37: 251-261.

Irache JM, Durrer C, Duchene D, Ponchel G (1994) In vitro study of lectin-latex conjugates for specific bioadhasion. J Contr Rel 31: 181-188.

Jozefowicz J, Jozefowicz M (1990) Interactions of biospecific functional polymers with blood proteins and cells. J Biomater Sci Polym Ed 1: 147-165.

Kataoka K, Okano T, Sakurai Y, Maruyama A, Tsuruta $T$ (1989) Controlled interactions of cells with multiphasestructured surfaces of block and graft copolymers. In: Multiphase Biomedical Materials (Tsuruta T, Nakajima A, eds) VSP, Urecht, p. 1.

Kataoka K, Miyazaki H, Okano T, Sakurai Y (1994) Sensitive glucose-induced change of the lower critical solution temperature of poly [ $N, N$-dimethylacrylamide-co3-(acrylamido) phenylboronic acid] in physiological saline. Macromolecules 27: 1061-1062.

Kijne W (1996) Functions of plant lectins. Chemt Biochem Mol Biol 6: 180-187.
Kulkarni GV, Lee W, Seth A, McCulloch CAG (1998) Role of mitochondrial membrane potential in concanavalin A-induced apoptosis in human fibroblasts. Exp Cell Res 245: 170-178.

Leist M, Wendel A (1998) Rat hepatocytes isolated from alcohol-induced fatty liver have an increased sensitivity to anoxic injury. J Hepatology 25: 948-959.

Herberman RB (1998)Lymphocyte-mediated cytotoxicity. In: The Lymphocyte (Marchalonis JJ, ed) Marcel Dekker Inc. New York, pp. 95-143.

Miyazaki H, Kikuchi A, Kitano S, Koyama Y, Okano T, Sakurai Y, Kataoka K (1993)0 Boronate-Containing Polymer as Novel Mitogen for Lymphocytes. Biochem Biophys Res Comm 195: 829-836.

Mulé JJ, Rosenberg SA (1985) The anti-tumor efficacy of lymphokine-activated killer cells and recombinant interleukin 2 in vivo. J Immunol 135: 646-654.

Mulé JJ, Shu S, Schwarz SL, Rosenberg SA (1984) Successfu 1 adoptive immunotherapy of established pulmonary metastases with LAK cells and recombinant IL-2. Science 225: 1487-1488.

Otsuka H, Nagasaki Y, Kataoka K (2000a) Surface characterization of functionalized polylactide through the coating with heterobifunctional poly(ethylene glycol)/ polylactide block copolymers. Biomacromolecules 1: 3948.

Otsuka H, Nagasaki Y, Kataoka K (2000b) Dynamic wettability study on the functionalized PEGylated layer on a polylactide surface constructed by the coating of aldehyde-ended poly(ethylene glycol) (PEG)/polylactide (PLA) block copolymer. Sci.Tech. Adv. Mater.1: 21-29.

Otsuka H, Uchimura E, Koshino H, Okano T, Kataoka K (2003) Anomalous Binding Profile of Phenylboronic Acid with $N$-Acetylneuraminic Acid (Neu5Ac) in Aqueous Solution with Varying pH. J Am.Chem Soc 125: 34933502.

Parhizgar A, Galleti PM, Jauregui HO (1987) Human endothelial cell attachment receptors. ASAIO Trans 33: 494-500.

Rasmussen JR, Stedronsky ER, Whitesides GM (1977a) Introduction, modification, and characterization of functional groups on the surface of low-density polyethylene film. J Am Chem Soc 99: 4736-4745.

Rasmussen JR, Bergbreiter DE, Whitesides GM (1977b) The question of delocalization in "anchored" ions with potential trishomoaromatic character. 1. Reduction of tricyclo[5.4.1.04,12]dodeca-2,5,8,10-tetraene as a route to the 10.pi.11C dianion. J Am Chem Soc 99: 4746-4756.

Sharon N, H. Lis H (1972) Lectins: cell-agglutinating and sugar-specific proteins. Science 177: 949-959.

Sharon N, H. Lis H (1998) Lectins: CarbohydrateSpecific Proteins That Mediate Cellular Recognition. Chem Rev 98: 637-674.

Uchimura E, Otsuka H, Okano T, Sakurai S, Kataoka K (2001) Totally synthetic polymer with lectin-like function: Induction of killer cells by the copolymer of 3acrylamidophenylboronic acid with N,NDimethylacrylamide. Biotech Bioeng 72: 307-314. 


\section{Discussion with Reviewers}

Reviewer I: Apparently the authors have not realised that the hydrophilic range of contact angles involves angles from $0^{\circ}-90^{\circ}$ and the hydrophobic range angles from $90^{\circ}$ to $180^{\circ}$. In Table 2 only contact angles $>110^{\circ}$ are listed i.e. the surface of the authors is very hydrophobic and shows a paradox behaviour in that at high $\mathrm{pH}$, when boronic acid dissociates and a more hydrophilic surface is expected due to the generated charges (and to increased binding of water molecules, as the authors correctly state), the authors observe an increase in contact angles i.e. an increase in hydrophobicity! This paradox effect is not considered by the authors. Thus the conclusion of the authors that "the proliferative response of lymphocytes was further emphasized by increased surface hydrophilicity" is not supported by the data.
Authors: Many studies have presented a theoretical and experimental comparison between two approaches to the contact angle measurement, i.e., the drop (water drop in air) and the captive bubble (air bubble in water) methods. In these studies, the general definitions have been shown for the contact angle measured by the drop and captive bubble methods; $\theta$ (degree) is the angle between the solid surface and the water drop or the air bubble interface measured inside the drop or bubble. is related to the actual contact angle $\theta_{\text {ac }}$ by:

$\theta=\theta_{\text {ac }}$ for a water drop

$\theta=180-\theta_{\text {ac }}$ for a captive bubble

Thus, in case of the captive bubble method, a higher contact angle $(\theta)$ corresponds to an increased hydrophilicity (smaller value of $\theta_{\mathrm{ac}}$ ). 